\title{
Goal-directed transfusion protocol via thrombelastography in patients with abdominal trauma: a retrospective study
}

Jianyi Yin ${ }^{1 \dagger}$, Zhenguo Zhao ${ }^{2 \dagger}$, Yousheng $\mathrm{Li}^{1 *}$, Jian Wang ${ }^{1}$, Danhua Yao ${ }^{1}$, Shaoyi Zhang ${ }^{1}$, Wenkui Yu ${ }^{1}$, Ning Li ${ }^{1}$ and Jieshou Li ${ }^{1}$

\begin{abstract}
Introduction: The optimal transfusion protocol remains unknown in the trauma setting. This retrospective cohort study aimed to determine if goal-directed transfusion protocol based on standard thrombelastography (TEG) is feasible and beneficial in patients with abdominal trauma.

Methods: Sixty adult patients with abdominal trauma who received 2 or more units of red blood cell transfusion within 24 hours of admission were studied. Patients managed with goal-directed transfusion protocol via TEG (goal-directed group) were compared to patients admitted before utilization of the protocol (control group).

Results: There were 29 patients in the goal-directed group and 31 in the control group. Baseline parameters were similar except for higher admission systolic blood pressure in the goal-directed group than the control group $(121.8 \pm 23.1 \mathrm{mmHg}$ vs $102.7 \pm 26.5 \mathrm{mmHg}, \mathrm{p}<0.01)$. At $24 \mathrm{~h}$, patients in the goal-directed group had shorter aPTT compared to patients in the control group $(39.2 \pm 16.3 \mathrm{~s}$ vs $58.6 \pm 36.6 \mathrm{~s}, \mathrm{p}=0.044)$. Administration of total blood products at $24 \mathrm{~h}$ appeared to be fewer in the goal-directed group than the control group (10.2 [7.0-43.1] U vs 14.8 [8.3-37.6]U, $p=0.28$, but this was not statistically significant. Subgroup analysis including patients with ISS $\geq 16$ showed that patients in the goal-directed group had significantly fewer consumption of total blood products than patients in the control group $(7[6.1,47.0] \cup$ vs $37.6[14.5,89.9] \mathrm{U}, \mathrm{p}=0.015)$. No differences were found in mortality at $28 \mathrm{~d}$, length of stay in intensive care unit and hospital between the two groups.

Conclusions: Goal-directed transfusion protocol via standard TEG was achievable in patients with abdominal trauma. The novel protocol, compared to conventional transfusion management, has the potential to decrease blood product utilization and prevent exacerbation of coagulation function.
\end{abstract}

Keywords: Transfusion, Thrombelastography, Trauma-induced coagulopathy, Abdominal trauma

\section{Introduction}

Uncontrollable hemorrhage is a major cause of early death in trauma patients [1]. Hemorrhage may occur due to direct injury, and is frequently complicated by coagulopathy $[2,3]$. Post-injury coagulopathy may exacerbate hemorrhage and contribute to poor outcome and an increased transfusion requirement $[4,5]$.

\footnotetext{
*Correspondence: liys@medmail.com.cn

${ }^{\dagger}$ Equal contributors

'Department of Surgery, Jinling Hospital, Nanjing University School of Medicine, Nanjing, China

Full list of author information is available at the end of the article
}

Blood transfusion is an essential component in trauma management. The goal of transfusion includes improvement of tissue oxygen delivery by replacing red blood cell, as well as prevention and correction of coagulation dysfunction by supplementing appropriate blood components. However, the optimal transfusion protocol for trauma patients remains unknown. In lack of guidance by rapid and comprehensive tools monitoring coagulation status, current transfusion protocols are unable to utilize blood products according to individual demands. As a consequence, these protocols are likely to lead to inappropriate and excessive administration of blood products, which is associated with increased burden of

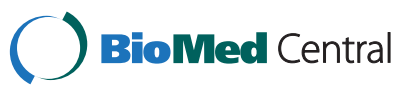

(c) 2014 Yin et al.; licensee BioMed Central Ltd. This is an Open Access article distributed under the terms of the Creative Commons Attribution License (http://creativecommons.org/licenses/by/2.0), which permits unrestricted use, distribution, and reproduction in any medium, provided the original work is properly credited. The Creative Commons Public Domain Dedication waiver (http://creativecommons.org/publicdomain/zero/1.0/) applies to the data made available in this article unless otherwise stated. 
blood product supply and risk of transfusion-related morbidity.

In recent years, viscoelastic hemostatic assays (VHA), including thrombelastography (TEG) and thrombelastometry, have been demonstrated to be ideal methods of monitoring coagulation function in trauma patients [6,7]. Furthermore, several studies have suggested the potential of VHA tests to guide component blood transfusion in a variety of patient groups [8-12]. In particular, a recent study by Kashuk et al. [13] showed that goaldirected transfusion based on rapid TEG was useful in managing trauma-induced coagulopathy, with the potential to reduce blood product administration in trauma patients.

A goal-directed transfusion protocol via TEG was implemented in our department since 2010 [14]. In the present study, we assessed the utilization of the protocol in abdominal trauma management by comparing outcomes of patients admitted before and after implementation of the protocol. We aimed to determine if the novel transfusion protocol could be successfully integrated in abdominal trauma management, and identify potential benefits of the protocol compared to conventional transfusion management.

\section{Materials and methods}

This cohort study analyzed the prospectively collected data of patients with abdominal trauma at Department of General Surgery, Jinling Hospital. The study was approved by the ethics committee of Jinling Hospital. Waiver of informed consent from patients was approved because of the observational nature of the study. Jinling Hospital is a tertiary teaching hospital in Nanjing, China. The Department of General Surgery is responsible for medical and surgical care of patients with abdominal trauma admitted to the emergency department (ED) of the hospital. At $E D$, a consulting surgeon judges the need for emergency laparotomy of the abdominal trauma patient. The patient is subsequently transferred to one of the two surgical intensive care units (SICU) of our department from ED if emergency laparotomy is not needed, or from operation room after emergency laparotomy. Non-operative care is provided by a team of surgeons and SICU specialists following previously published guidelines [15].

\section{Study population}

We searched the abdominal trauma database to identify potential patients between November 2008 and October 2012. Inclusion criteria were age older than 18 years, abdominal abbreviated injury scale $\geq 2$, and requirement of 2 or more units of red blood cell (RBC) transfusion within 24 hours of ED admission. Exclusion criteria included time interval between injury and ED admission $>24$ hours, major traumatic brain injury (head abbreviated injury scale $\geq 3$ ), end-staged liver disease, pregnancy, and history of anti-coagulation therapy in the latest 3 months.

All included patients were subsequently divided into 2 groups according to the time of admission. Patients between November 2008 and October 2010, who received conventional transfusion management, were assigned to the control group, whereas patients between November 2010 and October 2012, who were managed with the goal-directed transfusion protocol, were assigned to the goal-directed group.

\section{Transfusion protocol}

At ED, patients with abdominal trauma might receive preemptive transfusion of 2 units of RBC and 2-4 units of fresh frozen plasma (FFP) following initial fluid resuscitation when hemoglobin level was below $90 \mathrm{~g} / \mathrm{L}$ or showed active bleeding signs. Once the patient was planned to be transferred to our department, subsequent transfusion decisions were made by the treating surgeon or SICU specialist.

Patients in the control group received conventional transfusion management, which was based on individual experience and interpretation of conventional coagulation testing results of the treating surgeon or SICU specialist. RBC and FFP were delivered at a ratio of 1:1-1:2. Platelet and cryoprecipitate were administrated in selected cases.

The TEG 5000 thrombelastograph hemostasis analyzer system (Haemoscope Corporation, Niles, USA) was initially introduced to our department for monitoring post-operative coagulation function. The device enables point-of-care coagulation assay of whole blood at the patient's temperature. The device undergoes quality control daily according to the manufacturer's instructions. TEG analysis is carried out within 4 minutes of blood sample collection. The whole blood sample is placed in a manufacturer-supplied vial containing kaolin, and $0.35 \mathrm{ml}$ of the blood sample is added to a cup, followed by adjustment of the temperature setting to the patient's temperature. TEG assay is then started and stopped when reaching full tracing. A number of parameters are generated from the TEG tracing, each representing an aspect of hemostasis. The $\mathrm{R}$ value is the time from the beginning to the onset of clot formation, representing the activity of enzymatic clotting factors. The $\alpha$ angle is the angle between the tangent line and the horizontal line of the tracing, representing the activity of fibrinogen. The maximal amplitude (MA) is the overall clot strength, indicating the platelet activity.

Patients in the goal-directed group were managed with goal-directed transfusion protocol based on TEG results (Figure 1). The protocol was developed by a group of surgeons, SICU specialists, and transfusion specialists, and was introduced to all surgeons and SICU specialists of our 
department before its implementation in November 2010. The algorithm of the protocol was shown as hard copies in SICU, and two attending surgeons and two SICU specialists ensured utilization of the protocol as the leaders of abdominal trauma management. In specific, standard TEG test was ordered by the treating surgeon or SICU specialist when the patient with abdominal trauma was admitted to SICU, or had active bleeding at ED, operation room (OR), or SICU. Whole blood sample was transferred immediately to the SICU of our department, where it was analyzed. Results were fed back via in-hospital communication system to the treating surgeon or SICU specialist, who determined further transfusion management according to the goaldirected transfusion protocol. The goal-directed transfusion might occur at ED, OR, or SICU. Subsequent TEG tests were ordered until the patient had no active bleeding or coagulopathy.

\section{Data collection}

Data of all included patients from ED, SICU, OR, blood bank, and laboratory were linked. Demographic characteristics (age and gender), injury severity indices (injury mechanism, injured organs, injury severity score [ISS], abdominal abbreviated injury scale [AIS]) were collected. Administration of component blood products within 24 hours of ED admission was also recorded. Clinical and laboratory parameters of interest included vital signs (body temperature, heart rate, and systolic blood pressure), arterial blood gas results ( $\mathrm{pH}$, lactate, and base excess), blood cell counts (hemoglobin concentration, RBC count, and platelet count), albumin and calcium concentration, international normalized ratio (INR) and activated partial thromboplastin time (aPTT) at ED admission and $24 \mathrm{~h}$. TEG parameters ( $\mathrm{R}$ value, $\alpha$ angle, and MA value) of the first TEG test and the follow-up one between 24-48 hours after the first one were collected from patients in the

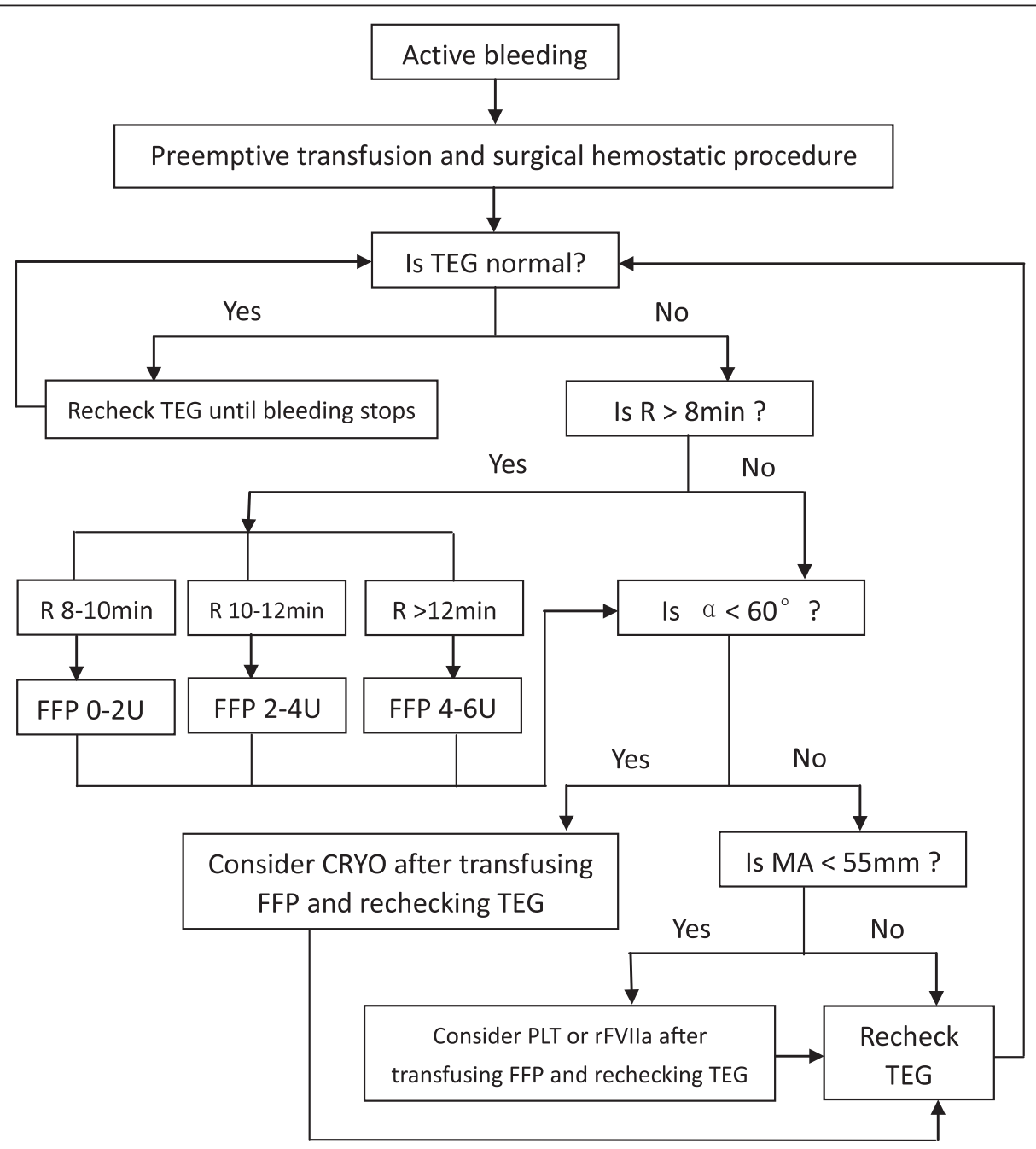

Figure 1 Goal-directed transfusion protocol via TEG. 
goal-directed group. In addition, mortality at $28 \mathrm{~d}$, length of stay in ICU and hospital were noted. An independent investigator checked the accuracy of collected data before analysis.

\section{Statistical analysis}

We used SPSS software (v19.0 for Windows, IBM Corporation, USA) for statistical analysis. Normality of distribution was analyzed by Kolmogorov-Smirnov test. Continuous variables with normal distribution and skewed distribution were analyzed using Student's $t$ test and Mann-Whitney $u$ test, respectively. Categorical variables were analyzed using chi-square test. Significance was considered as $p<0.05$.

\section{Results}

\section{Patient characteristics}

A total of 150 patients with abdominal trauma were admitted between November 2008 and October 2012, of whom 98 met the inclusion criteria. Thirty-eight patients were excluded due to prolonged time interval between injury and ED admission $(n=36)$, end-staged liver disease $(\mathrm{n}=1)$, and major traumatic brain injury $(\mathrm{n}=1)$, leaving 60 patients for final analysis (Figure 2).
There were 31 patients in the control group and 29 in the goal-directed group. The two groups were comparable in terms of age and gender. The control group and the goal-directed group had similar ISS $(14.3 \pm 5.7$ vs $16.2 \pm 8.0, \mathrm{p}=0.28)$ and abdominal AIS $(3.1 \pm 0.7$ vs $3.1 \pm 0.9, \mathrm{p}=0.86$ ). There were, however, more frequent patients with pancreatic injury in the goal-directed group than the control group ( $44.8 \%$ vs $16.1 \%, \mathrm{p}=0.015)$. All but 3 patients ( 2 in the control group and 1 in the goaldirected group) underwent emergency operation for control of intra-abdominal bleeding or repair of intraabdominal organ injury (Table 1 ).

\section{Administration of blood products}

Administration of blood products within 24 hours of ED admission was presented in Table 2. No significant differences were found in the proportion of patients receiving FFP $(100 \%$ vs $96.8 \%, \mathrm{p}=1.0)$, platelet $(13.8 \%$ vs $29.0 \%$, $\mathrm{p}=0.15)$, and cryoprecipitate $(24.1 \%$ vs $29.0 \%, \mathrm{p}=0.67)$ between the goal-directed group and the control group. Administration of RBC, FFP, platelet, cryoprecipitate, and total blood products was fewer in the goal-directed group than the control group, but this did not reach

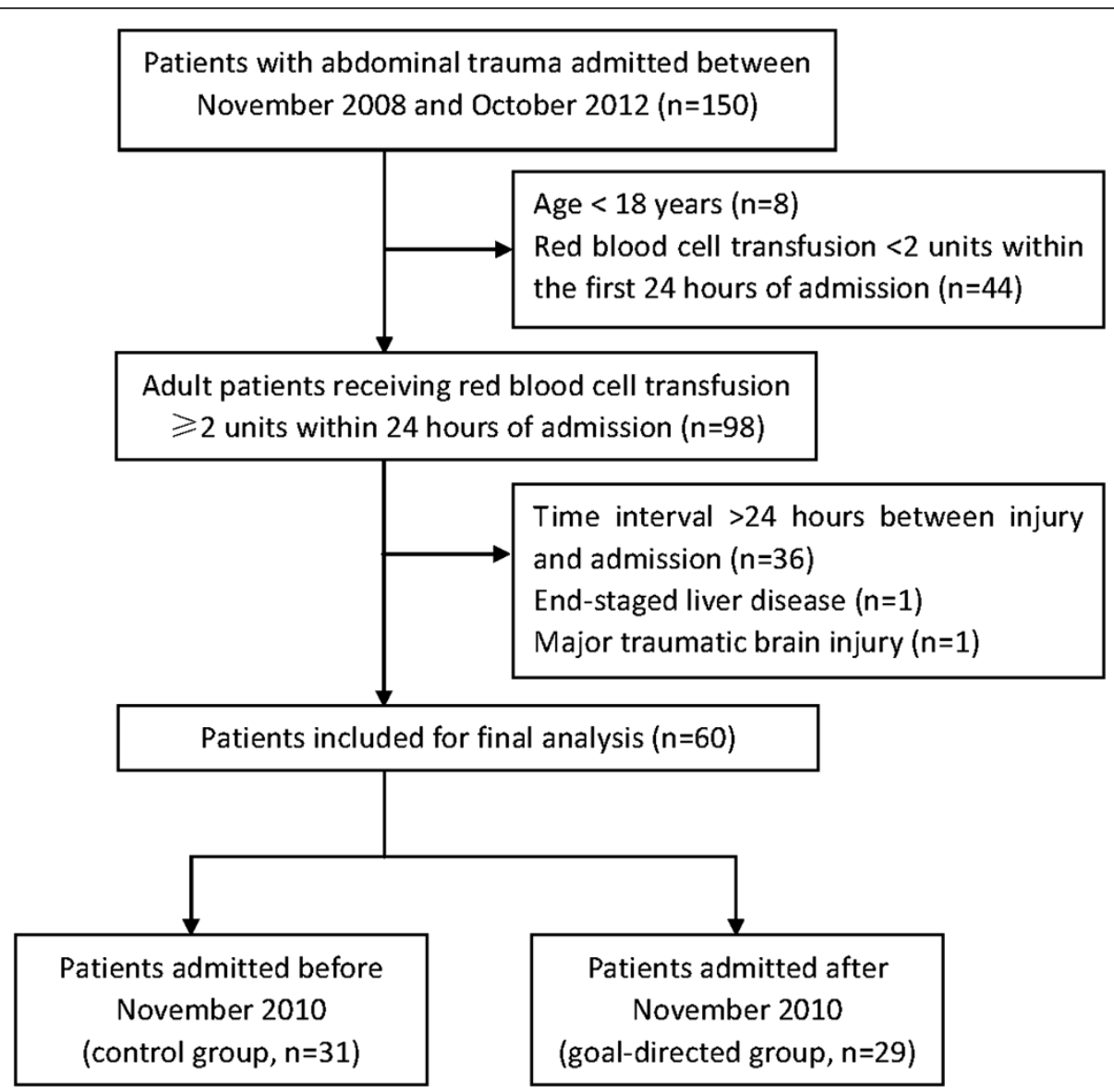

Figure 2 Flowchart showing patient inclusion and exclusion. 
Table 1 Patient characteristics ${ }^{\mathrm{a}}$

\begin{tabular}{|c|c|c|c|c|}
\hline & Overall $(n=60)$ & Control group $(n=31)$ & Goal-directed group $(n=29)$ & $\mathbf{p}$ \\
\hline Age (year) & $41.7 \pm 14.2$ & $42.8 \pm 15.6$ & $40.5 \pm 12.8$ & 0.53 \\
\hline \multicolumn{5}{|l|}{ Gender } \\
\hline Male & 49(81.7) & 26(83.9) & 23(79.3) & \multirow[t]{2}{*}{0.65} \\
\hline Female & $11(18.3)$ & $5(16.1)$ & $6(20.7)$ & \\
\hline \multicolumn{5}{|l|}{ Mechanism of injury } \\
\hline Blunt & $50(83.3)$ & $27(87.1)$ & 23(79.3) & \multirow[t]{2}{*}{0.64} \\
\hline Penetrating & $10(16.7)$ & $4(12.9)$ & $6(20.7)$ & \\
\hline ISS & $15.2 \pm 6.9$ & $14.3 \pm 5.7$ & $16.2 \pm 8.0$ & 0.28 \\
\hline Abdominal AIS & $3.1 \pm 0.8$ & $3.1 \pm 0.7$ & $3.1 \pm 0.9$ & $0.86^{b}$ \\
\hline \multicolumn{5}{|c|}{ Involved abdominal organ } \\
\hline Spleen & $24(40.0)$ & $15(48.4)$ & $9(31.0)$ & 0.17 \\
\hline Liver & $14(23.3)$ & $9(29.0)$ & $5(17.2)$ & 0.28 \\
\hline Pancreas & 18(30.0) & $5(16.1)$ & 13(44.8) & 0.015 \\
\hline Vessel & $5(8.3)$ & $4(12.9)$ & $1(3.4)$ & 0.39 \\
\hline Stomach & $4(6.7)$ & $1(3.2)$ & $3(10.3)$ & 0.35 \\
\hline Duodenum & $6(10.0)$ & $4(12.9)$ & $2(6.9)$ & 0.73 \\
\hline Intestine & $12(20)$ & $5(16.1)$ & $7(24.1)$ & 0.44 \\
\hline Colon & $14(23.3)$ & $6(19.4)$ & $8(27.6)$ & 0.45 \\
\hline Rectum & $2(3.3)$ & $1(3.2)$ & $1(3.4)$ & 1.00 \\
\hline Emergency operation & $57(95)$ & 29(93.5) & 28(96.6) & 1.00 \\
\hline ICU stay (day) & $10.1 \pm 9.2$ & $8.1 \pm 5.5$ & $12.2 \pm 11.8$ & $0.28^{b}$ \\
\hline Hospital stay (day) & $13.4 \pm 10.0$ & $11.3 \pm 6.2$ & $15.6 \pm 12.7$ & 0.10 \\
\hline Mortality at $28 \mathrm{~d}$ & $5(8.3)$ & $2(6.5)$ & $3(10.3)$ & 0.94 \\
\hline
\end{tabular}

${ }^{\mathrm{a}}$ Data are presented as mean \pm SD or number(\%).

${ }^{\mathrm{b}}$ Mann-Whitney u test.

ISS, injury severity score; AIS, abbreviated injury scale, ICU: intensive care unit.

statistical significance. We further performed subgroup analysis including patients with ISS $\geq 16$. The results showed that patients in the goal-directed group $(n=16)$ had significantly fewer consumption of RBC $(4[3,11.5] \mathrm{U}$ vs $14[7.5,32] \mathrm{U}, \mathrm{p}<0.01)$, FFP $(4[2.9,9.8] \mathrm{U}$ vs $10.5[5.6$, 15.7]U, $\mathrm{p}=0.036)$ and total blood products $(7[6.1,47.0]$ $\mathrm{U}$ vs $37.6[14.5,89.9] \mathrm{U}, \mathrm{p}=0.015)$ than patients in the control group $(n=13)$, whereas consumption of platelet and cryoprecipitate was not significantly different. Furthermore, the cost of total blood product appeared to be lower in the goal-directed group than the control group (\$227.5[152.9, 1221.7] vs \$329.0 [197.2, 2904.8]), but this was not significantly different $(\mathrm{p}=0.156)$.

\section{Clinical and laboratory parameters}

Clinical and laboratory parameters of interest at ED admission and $24 \mathrm{~h}$ were summarized in Table 3 . Patients in the goal-directed group had significantly higher systolic blood pressure at ED admission $(121.8 \pm 23.1 \mathrm{mmHg}$ vs $102.7 \pm 26.5 \mathrm{mmHg}, \mathrm{p}=0.005)$ and lower $\mathrm{pH}(7.39 \pm 0.06$

Table 2 Administration of blood products at $24 h^{a}$

\begin{tabular}{|c|c|c|c|c|c|c|c|}
\hline & \multicolumn{3}{|c|}{ Control group $(n=31)$} & \multicolumn{3}{|c|}{ Goal-directed group $(n=29)$} & \multirow{2}{*}{$\mathrm{p}$} \\
\hline & Number & Median & IQR & Number & Median & IQR & \\
\hline $\mathrm{RBC}(U)$ & 31 & 6.5 & $4-14$ & 29 & 5 & $3-13$ & 0.22 \\
\hline FFP $(U)$ & 30 & 6.1 & $4-10.7$ & 29 & 5.7 & $3.4-10$ & 0.54 \\
\hline PLT (U) & 9 & 0 & $0-10$ & 4 & 0 & $0-0$ & 0.15 \\
\hline CRYO (U) & 9 & 0 & $0-10$ & 7 & 0 & $0-5$ & 0.68 \\
\hline Total (U) & 31 & 14.8 & 8.3-37.6 & 29 & 10.2 & 7.0-43.1 & 0.28 \\
\hline
\end{tabular}

${ }^{\mathrm{a}}$ Data were analyzed using Mann-Whitney u test.

RBC: red blood cell; FFP: fresh frozen plasma; PLT: platelet; CRYO: cryoprecipitate; IQR: interquartile range. 
Table 3 Clinical and laboratory parameters

\begin{tabular}{|c|c|c|c|c|c|c|c|c|c|c|}
\hline & \multicolumn{5}{|c|}{ At ED admission } & \multicolumn{5}{|c|}{ At $24 \mathrm{~h}$} \\
\hline & \multicolumn{2}{|c|}{ Control group $(n=31)$} & \multicolumn{2}{|c|}{ Goal-directed group $(n=29)$} & \multirow{2}{*}{ p } & \multicolumn{2}{|c|}{ Control group $(n=31)$} & \multicolumn{2}{|c|}{ Goal-directed group $(n=28)$} & \multirow[b]{2}{*}{$p$} \\
\hline & Number & Mean \pm SD & Number & Mean \pm SD & & Number & Mean \pm SD & Number & Mean \pm SD & \\
\hline Temperature $\left({ }^{\circ} \mathrm{C}\right)$ & 31 & $36.4 \pm 0.3$ & 29 & $36.4 \pm 0.3$ & 0.98 & 31 & $37.2 \pm 0.7$ & 28 & $37.2 \pm 0.6$ & 0.84 \\
\hline $\mathrm{HR}$ (/min) & 31 & $100.3 \pm 19.5$ & 28 & $91.8 \pm 18.7$ & 0.09 & 31 & $101.4 \pm 18.6$ & 28 & $96.9 \pm 18.3$ & 0.35 \\
\hline SBP $(\mathrm{mmHg})$ & 31 & $102.7 \pm 26.5$ & 28 & $121.8 \pm 23.1$ & 0.005 & 31 & $122.4 \pm 16.8$ & 28 & $122.6 \pm 14.7$ & 0.97 \\
\hline $\mathrm{Hb}(\mathrm{g} / \mathrm{L})$ & 30 & $121.1 \pm 20.6$ & 28 & $122.5 \pm 24.0$ & 0.82 & 31 & $105.5 \pm 15.2$ & 27 & $106.6 \pm 18.9$ & 0.8 \\
\hline $\operatorname{RBC}\left(\times 10^{12} / \mathrm{L}\right)$ & 30 & $3.9 \pm 0.6$ & 27 & $4.1 \pm 0.7$ & 0.27 & 31 & $3.4 \pm 0.5$ & 27 & $3.5 \pm 0.6$ & 0.69 \\
\hline PLT $\left(\times 10^{9} / \mathrm{L}\right)$ & 30 & $186.2 \pm 52.9$ & 28 & $181.1 \pm 59.0$ & 0.73 & 31 & $113.0 \pm 45.1$ & 27 & $116.6 \pm 47.7$ & 0.77 \\
\hline $\mathrm{pH}$ & 16 & $7.38 \pm 0.05$ & 14 & $7.38 \pm 0.04$ & 0.66 & 25 & $7.41 \pm 0.04$ & 27 & $7.39 \pm 0.06$ & 0.048 \\
\hline Lactate (mmol/L) & 16 & $2.8 \pm 1.5$ & 14 & $3.1 \pm 2.4$ & 0.68 & 25 & $2.6 \pm 1.7$ & 27 & $2.1 \pm 1.4$ & $0.18^{a}$ \\
\hline $\mathrm{BE}(\mathrm{mmol} / \mathrm{L})$ & 16 & $(-3.9) \pm 3.4$ & 14 & $(-3.0) \pm 3.5$ & 0.48 & 25 & $(-2.7) \pm 4.6$ & 27 & $(-2.4) \pm 2.5$ & 0.75 \\
\hline Albumin (g/L) & 28 & $38.3 \pm 6.1$ & 28 & $38.1 \pm 7.3$ & 0.92 & 31 & $33.2 \pm 5.8$ & 27 & $33.6 \pm 4.5$ & 0.79 \\
\hline Calcium (mmol/L) & 25 & $2.1 \pm 0.2$ & 27 & $2.1 \pm 0.2$ & 0.91 & 31 & $2.0 \pm 0.2$ & 27 & $2.0 \pm 0.2$ & 0.28 \\
\hline INR & 27 & $1.1 \pm 0.2$ & 28 & $1.1 \pm 0.1$ & 0.73 & 26 & $1.2 \pm 0.2$ & 24 & $1.2 \pm 0.2$ & 0.97 \\
\hline aPTT (s) & 27 & $28.4 \pm 6.4$ & 28 & $25.7 \pm 4.8$ & 0.09 & 26 & $58.6 \pm 36.6$ & 24 & $39.2 \pm 16.3$ & $0.044^{\mathrm{a}}$ \\
\hline
\end{tabular}

${ }^{\mathrm{a} M a n n-W h i t n e y ~ u ~ t e s t . ~}$

vs $7.41 \pm 0.04, \mathrm{p}=0.048)$ at $24 \mathrm{~h}$ than patients in the control group. In addition, aPTT at $24 \mathrm{~h}$ was significantly shorter in the goal-directed group compared to the control group $(39.2 \pm 16.3 \mathrm{~s}$ vs $58.6 \pm 36.6 \mathrm{~s}, \mathrm{p}=0.044)$, while admission aPTT was similar $(25.7 \pm 4.8 \mathrm{~s}$ vs $28.4 \pm 6.4 \mathrm{~s}$, $\mathrm{p}=0.09$ ). No significant differences were observed in other parameters between the two groups.

The first TEG test in the goal-directed group showed $\mathrm{R}$ value of $10.1 \pm 4.7 \mathrm{~min}, \alpha$ angle of $44.1 \pm 16.1$, and MA value of $50.0 \pm 12.1$. A follow-up TEG test between 24-48 hours after the first TEG test was available from 21 patients, with improved $R$ value of $8.5 \pm 4.7 \mathrm{~min}(\mathrm{p}=0.037)$, $\alpha$ angle of $51.1 \pm 11.5(\mathrm{p}<0.001)$, and MA value of $52.0 \pm$ $13.3(\mathrm{p}=0.11)$.

\section{Clinical outcomes}

There were 3 deaths ( 1 for exsanguination at $24 \mathrm{~h}, 1$ for multiple organ dysfunction at 72 h, 1 for coagulopathy at $14 \mathrm{~d})$ in the goal-directed group and 2 deaths for coagulopathy ( 1 at $48 \mathrm{~h}$ and 1 at $72 \mathrm{~h}$ ) in the control group. No significant differences were found in mortality at $28 \mathrm{~d}$, length of stay in ICU and hospital between the two groups.

\section{Discussion}

This cohort study showed that goal-directed transfusion protocol via TEG was applicable in patients with abdominal trauma, and was associated with a trend towards fewer blood product utilization and better coagulation profile at $24 \mathrm{~h}$ compared to conventional transfusion management. The results support the use of TEG in guiding transfusion management in patients with abdominal trauma.
First, this study provides supplemental evidence for using TEG to guide transfusion management in the trauma setting. TEG has been shown to be helpful in detecting post-injury coagulopathy and directing transfusion management in patients with severe multiple trauma [13], but the use of TEG in patients with lower injury severity has not been thoroughly investigated, which may be due to the relatively low incidence of coagulopathy in moderately injured patients [2]. In this study, the majority of included patients sustained moderate abdominal injury, as suggested by mean ISS of 15.2 and mean abdominal AIS of 3.1. Despite the relatively low injury severity, our patients were still exposed to risk of coagulation dysfunction, as suggested by aggravation of INR and aPTT during the first 24 hours of ED admission. The exacerbation of coagulation function might be associated with primary injury, second hit of operation, blood loss, and massive fluid resuscitation [16]. We found that TEG had reasonable value in monitoring coagulation function in these patients, and goal-directed transfusion protocol via TEG could be successfully implemented.

Second, our results showed that goal-directed transfusion protocol via TEG had the potential to reduce administration of component blood products. Although not statistically significant, patients managed with goaldirected transfusion protocol received fewer component blood products, especially RBC and FFP than patients receiving conventional transfusion management. In subgroup analysis including patients with ISS $\geq 16$, we showed that goal-directed transfusion protocol led to significant reduction in administration of $\mathrm{RBC}$, FFP, and total blood 
products. These results are consistent with the findings of several previous studies $[8,11,13]$. Moreover, we found that the reduction in blood product administration did not compromise perfusion status and oxygen delivery capacity, as evidenced by similar lactate level, hemoglobin concentration, and RBC count at $24 \mathrm{~h}$ between the two patient groups. The reduction of blood product administration is important in two aspects. First, it relieves the burden of blood product supply, and may have the potential to decrease the cost of blood products for patients. Second, it is likely to lower transfusion-related morbidity, such as coagulopathy, transfusion-related acute lung injury, and infection [17]. However, these findings must be interpreted with caution given the small sample size of the study and subgroup analysis.

Third, goal-directed transfusion protocol appears to be better than conventional transfusion management in preventing coagulation function exacerbation after transfusion. In recent years, there is improving understanding in acute traumatic coagulopathy (ATC), which is resulted from tissue injury and hypoperfusion due to trauma. Subsequent medical interventions, such as massive transfusion, may further exacerbate coagulation dysfunction and lead to trauma-induced coagulopathy (TIC) [18]. In this study, we observed that patients in the goal-directed group had better coagulation profile at $24 \mathrm{~h}$, as indicated by shorter aPTT, than patients in the control group. Furthermore, the TEG parameters were significantly improved in patients managed with goal-directed transfusion protocol. There are two possible explanations for these findings. First, goal-directed transfusion protocol could prevent coagulation function worsening through supplementing appropriate blood component according to individual requirement. Second, the reduction of blood product utilization, as a result of the use of goal-directed transfusion protocol, might lower the risk of TIC secondary to massive transfusion. However, these findings needed to be interpreted carefully, since aPTT can represent only part of the coagulation system, and is affected by multiple factors [19]. Moreover, although aPTT results were available in more than $83.3 \%$ and follow-up TEG results were available in $72.4 \%$ of patients, missing data might reduce the power of the results.

The present study did not observe benefits of goaldirected transfusion protocol on mortality, which is not surprising because of the small sample size and moderate injury severity in overall. The previous study by Kashuk et al. [13] did not conclude the effect of goal-directed transfusion management on mortality either, because of incomparable injury severity between the patient groups. Considering the potential of goal-directed transfusion protocol in decreasing transfusion-related morbidity and correcting post-injury coagulopathy, it would be justified to infer that goal-directed transfusion protocol might improve mortality of trauma patients. Further studies are needed to investigate this issue.

Several limitations are worth considering when interpreting the results of this study. First, this is a retrospective study with small sample size. Due to the retrospective nature, we could not achieve two identical patient groups, as manifested by different admission systolic blood pressure between the two groups. Second, we did not abandon conventional coagulation tests after implementation of TEG. Therefore, the influence of conventional coagulation testing results on goal-directed transfusion management could not be eliminated and should be taken into consideration. Third, we were using standard TEG to guide transfusion, rather than rapid TEG. Moreover, we were not able to perform "baseline TEG", which was shown to be important for patients receiving TEG monitoring, since we were studying trauma patients in this study. Finally, this single institution experience may not be generalized because of different strategies in resuscitation, transfusion, and operation between trauma centers.

\section{Conclusions}

In summary, the present study showed that goal-directed transfusion protocol via TEG was feasible in patients with abdominal trauma, and was better than conventional transfusion management in reducing blood product utilization and preventing coagulation function exacerbation. The results are in favor of implementation of goal-directed transfusion protocol in trauma patients. Further studies are needed to confirm the benefits of the novel transfusion strategy in the trauma setting.

\section{Abbreviations}

VHA: Viscoelastic hemostatic assay; TEG: Thrombelastography; ED: Emergency department; SICU: Surgical intensive care unit; RBC: Red blood cell; FFP: Fresh frozen plasma; OR: Operation room; ISS: Injury severity score; AIS: Abdominal abbreviated injury scale; INR: International normalized ratio; aPTT: activated partial thromboplastin time.

\section{Competing interests}

The authors declare that they have no competing interests.

\section{Authors' contributions}

$J Y$ and $Z Z$ initiated the idea, carried out the study, and drafted the manuscript. JW, DY, and SZ helped collect and analyze data. YL and WY participated in the design of the study. NL and $J$ participated in the coordination of the study and helped to draft the manuscript. All authors read and approved the final manuscript.

\section{Authors' information}

Jianyi Yin and Zhenguo Zhao are joint first authors.

\section{Author details}

'Department of Surgery, Jinling Hospital, Nanjing University School of Medicine, Nanjing, China. Department of General Surgery, The Affiliated Jiangyin Hospital of Southeast University Medical College, Jiangyin, China.

Received: 28 January 2014 Accepted: 10 April 2014

Published: 15 April 2014 


\section{References}

1. Sauaia A, Moore FA, Moore EE, Moser KS, Brennan R, Read RA, Pons PT: Epidemiology of trauma deaths: a reassessment. J Trauma 1995, 38:185-193.

2. Brohi K, Singh J, Heron M, Coats T: Acute traumatic coagulopathy. J Trauma 2003, 54:1127-1130.

3. MacLeod JB, Lynn M, McKenney MG, Cohn SM, Murtha M: Early coagulopathy predicts mortality in trauma. J Trauma 2003, 55:39-44.

4. Maegele $M$, Lefering $R$, Yucel $N$, Tjardes T, Rixen D, Paffrath T, Simanski C, Neugebauer E, Bouillon B: Early coagulopathy in multiple injury: an analysis from the German Trauma Registry on 8724 patients. Injury 2007, 38:298-304.

5. Brohi K, Cohen MJ, Ganter MT, Matthay MA, Mackersie RC, Pittet JF: Acute traumatic coagulopathy: initiated by hypoperfusion: modulated through the protein C pathway? Ann Surg 2007, 245:812-818.

6. Holcomb JB, Minei KM, Scerbo ML, Radwan ZA, Wade CE, Kozar RA, Gill BS, Albarado R, McNutt MK, Khan S, Adams PR, McCarthy JJ, Cotton BA: Admission rapid thrombelastography can replace conventional coagulation tests in the emergency department: experience with 1974 consecutive trauma patients. Ann Surg 2012, 256:476-486.

7. Tauber H, Innerhofer P, Breitkopf R, Westermann I, Beer R, El Attal R, Strasak A, Mittermayr M: Prevalence and impact of abnormal ROTEM(R) assays in severe blunt trauma: results of the 'Diagnosis and Treatment of TraumaInduced Coagulopathy (DIA-TRE-TIC) study'. Br J Anaesth 2011, 107:378-387.

8. Johansson PI: Goal-directed hemostatic resuscitation for massively bleeding patients: the Copenhagen concept. Transfus Apher Sci 2010, 43:401-405.

9. Wang SC, Shieh JF, Chang KY, Chu YC, Liu CS, Loong CC, Chan KH, Mandell S, Tsou MY: Thromboelastography-guided transfusion decreases intraoperative blood transfusion during orthotopic liver transplantation: randomized clinical trial. Transplant Proc 2010, 42:2590-2593.

10. Schochl H, Nienaber U, Hofer G, Voelckel W, Jambor C, Scharbert G, Kozek-Langenecker S, Solomon C: Goal-directed coagulation management of major trauma patients using thromboelastometry (ROTEM)-guided administration of fibrinogen concentrate and prothrombin complex concentrate. Crit Care 2010, 14:R55.

11. Shore-Lesserson L, Manspeizer HE, DePerio M, Francis S, Vela-Cantos F, Ergin MA: Thromboelastography-guided transfusion algorithm reduces transfusions in complex cardiac surgery. Anesth Analg 1999, 88:312-319.

12. Johansson PI, Stensballe J: Effect of haemostatic control resuscitation on mortality in massively bleeding patients: a before and after study. Vox Sang 2009, 96:111-118.

13. Kashuk JL, Moore EE, Wohlauer M, Johnson JL, Pezold M, Lawrence J, Biffl WL, Burlew CC, Barnett C, Sawyer M, Sauaia A: Initial experiences with point-of-care rapid thrombelastography for management of life-threatening postinjury coagulopathy. Transfusion 2012, 52:23-33.

14. Yao D, Li Y, Wang J, Yu W, Li N, Li J: Effects of recombinant activated factor VIla on abdominal trauma patients. Blood Coagul Fibrinolysis 2014, 25:33-38.

15. Stassen NA, Bhullar I, Cheng JD, Crandall M, Friese R, Guillamondegui O, Jawa R, Maung A, Rohs TJ Jr, Sangosanya A, Schuster K, Seamon M, Tchorz KM, Zarzuar BL, Kerwin A, Eastern Association for the Surgery of Trauma: Nonoperative management of blunt hepatic injury: an Eastern Association for the Surgery of Trauma practice management guideline. J Trauma Acute Care Surg 2012, 73:S288-S293.

16. Frith D, Brohi K: The pathophysiology of trauma-induced coagulopathy. Curr Opin Crit Care 2012, 18:631-636.

17. Sihler KC, Napolitano LM: Massive transfusion: new insights. Chest 2009 , 136:1654-1667.

18. Frith D, Davenport R, Brohi K: Acute traumatic coagulopathy. Curr Opin Anaesthesiol 2012, 25:229-234.

19. Brohi K, Cohen MJ, Davenport RA: Acute coagulopathy of trauma: mechanism, identification and effect. Curr Opin Crit Care 2007, 13:680-685.

doi:10.1186/1749-7922-9-28

Cite this article as: Yin et al:: Goal-directed transfusion protocol via thrombelastography in patients with abdominal trauma: a retrospective study. World Journal of Emergency Surgery 2014 9:28.

\section{Submit your next manuscript to BioMed Central and take full advantage of:}

- Convenient online submission

- Thorough peer review

- No space constraints or color figure charges

- Immediate publication on acceptance

- Inclusion in PubMed, CAS, Scopus and Google Scholar

- Research which is freely available for redistribution

Submit your manuscript at www.biomedcentral.com/submit
C Biomed Central 